\title{
RNAi tackles a sexually transmitted disease
}

\author{
Patricia Cristofaro \& Bharat Ramratnam
}

RNA therapy is effective against Herpes simplex virus-2 in mice.

Herpes simplex virus-2 (HSV-2) infection has reached epidemic proportions, with seroprevalence rates ranging from $60-80 \%$ in young adults in resource-poor areas of the world. In the United States, nearly 45-million Americans are infected with HSV-2, now the most common cause of genital ulcerations, which increase the risk of acquiring other sexually transmitted infections. Thus, there is an urgent need for preventative HSV-2 therapies. Writing in Nature, Palliser et al. ${ }^{1}$ report that the process of RNA interference (RNAi) can be harnessed to prevent vaginal transmission of HSV-2 in a rodent model. This study is the first to show that vaginal administration of an siRNA therapeutic can be used prophylactically against a sexually transmitted viral infection both pre- and post-exposure.

RNAi refers to gene silencing mediated by single- or double-stranded RNA. Originally identified in Caenorhabditis elegans, RNAi was applied to mammalian systems in experiments demonstrating that gene expression in mammalian cell lines can be silenced by transfection of short (21-23 nucleotides), double-stranded RNA, termed short-interfering RNA ( $s i R N A)^{2}$. RNAi has quickly become an indispensable laboratory tool for selective knockdown of protein expression in cultured cells; moreover, the therapeutic potential of siRNA, delivered intravenously or locally, is under active investigation (Fig. 1).

Mucosal surfaces, the gateway of most infectious diseases, appear to be an ideal target for RNAi. Intranasal or intratracheal administration of siRNA targeting viral genes has proven effective in abrogating infection from respiratory syncytial virus ${ }^{3,4}$, parainfluenza virus ${ }^{3}, \mathrm{SARS}^{5}$ and influenza A, including avian strains of H5N1 (refs. 6,7), in relevant animal models.

Vaginal epithelium represents a new frontier in RNAi therapy. Palliser et al. have shown that mice can be protected from

Patricia Cristofaro \& Bharat Ramratnam are at the Laboratory of Retrovirology, Division of Infectious Diseases, Department of Medicine, Brown Medical School, Providence, Rhode Island 02878, USA.

e-mail:bramratnam@lifespan.org lethal HSV-2 infection by vaginal application of siRNA targeting two viral genes: $U L 29$, encoding a DNA-binding protein; and UL27, encoding envelope glycoprotein $B$. The products of these genes are essential for productive viral infections: UL29 codes for the protein ICP8, which is required for DNA replication, while UL27 codes for glycoprotein $\mathrm{B}$, which attaches the viron to the host cell surface.

Palliser et al. treated mice with antiviral or control siRNA $2 \mathrm{~h}$ before and $4 \mathrm{~h}$ after vaginal virus challenge and monitored disease severity and survival for fifteen days. Although all control mice shed virus on day 6 , no virus was detected in $70 \%$ of the mice treated with UL2 9 siRNA and $50 \%$ of the mice treated with UL27-siRNA. The investigators also assessed the antiviral efficacy of post-exposure treatment by administering siRNA 3 and $6 \mathrm{~h}$ after vaginal virus challenge. Only a combination of UL 27 and UL29 siRNA, but neither siRNA alone, protected animals from HSV-2 infection in this scenario.
Despite these promising results, a number of challenges confront the clinical development of siRNA as an antiviral vaginal microbicide. siRNA microbicides must be effective for a long time after a single application, must be able to neutralize a diversity of viral strains, must not induce mucosal inflammation and must cause minimal side effects.

In their studies, Palliser et al. timed vaginal virus challenges $2-6 \mathrm{~h}$ before or after siRNA administration. It is unclear whether patient compliance will be sufficiently high if microbicidal compounds are to be selfadministered according to impending sexual activity. The investigators pointed out that they used unmodified siRNA and that it may be possible to prolong mucosal gene silencing by using more stable siRNA analogs, an area of intense investigation. Palliser et al. used lipid reagents for the delivery of siRNA. Formulation of siRNA in vehicles such as sustained-release capsules or nano/ microparticles will be critical for future clinical use.

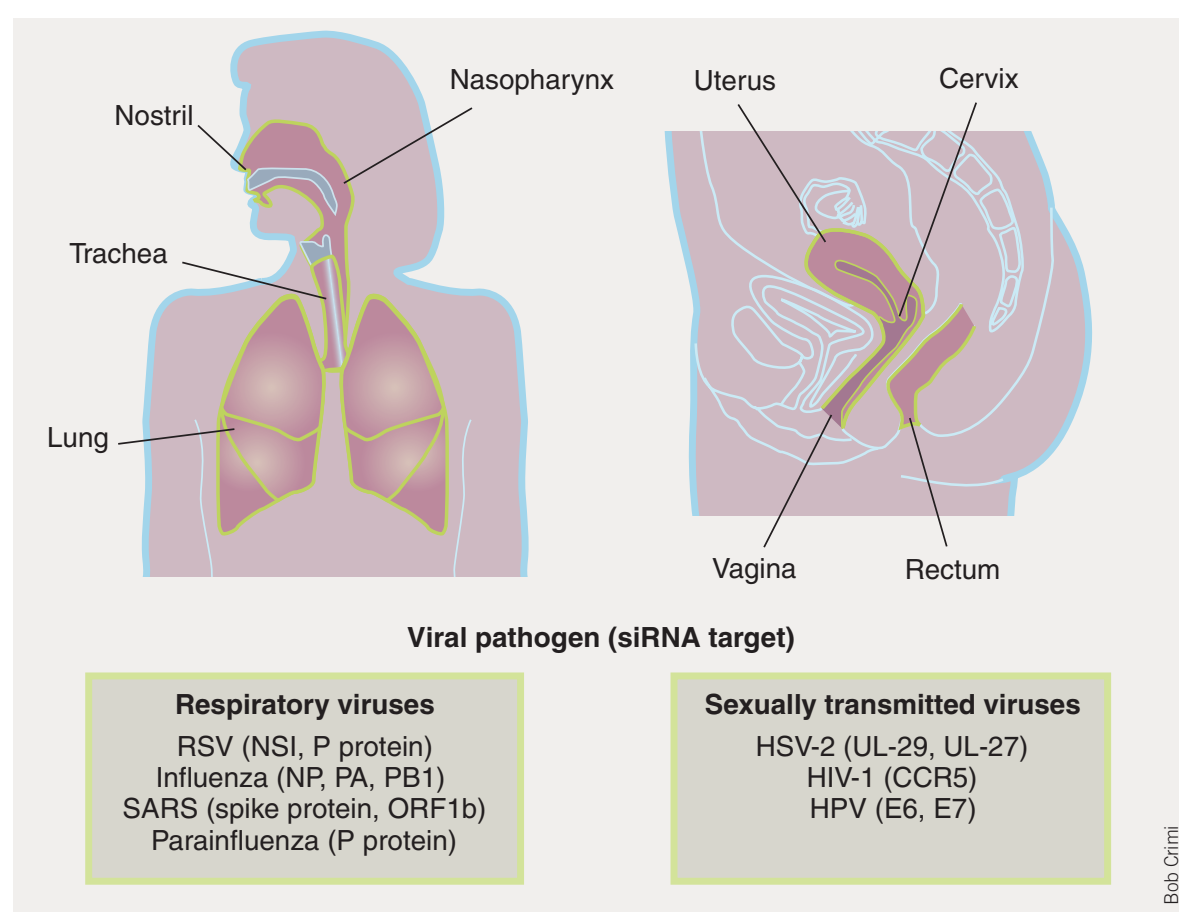

Figure 1 Antiviral siRNA therapeutics have previously been applied in treating respiratory infections (left). Palliser et al. have now applied the same technology to treatment of a sexually transmitted disease (right). 
An additional challenge is the choice of viral gene target. Ideally, a target must be conserved in a wide range of viral clinical isolates and must not be subject to mutational pressures in vivo. The development of viral resistance to siRNA has already been observed with hepatitis $\mathrm{C}^{8}$, poliovirus ${ }^{9}$ and $\mathrm{HIV}-1^{10}$. Mutational pressure can perhaps be countered by a cocktail of siRNAs directed at multiple viral genes. Alternatively, host proteins essential for virus entry or replication, such as the HIV-1 chemokine coreceptor CCR5, may also serve as useful siRNA targets.

Palliser et al. found no mucosal toxicity associated with siRNA administration. Similar studies of chronic use of siRNA will be needed, as long-term dosing is more likely to lead to nonspecific interferon responses and unexpected consequences of gene silenc- ing. Numerous investigations have revealed that siRNA can degrade targets that have less than $100 \%$ sequence complementarity. Thus, siRNA administration may lead to side effects through unintentional knockdown of host proteins.

Nevertheless, the work of Palliser et al. demonstrates the enormous potential of RNAi in the prevention and control of viral infections. The greatest advantage of this technology is the speed with which genomic information can potentially be translated into effective therapeutics. siRNA-based therapeutics can be rationally designed as fast as genomic information is made available. They can then be rapidly screened for efficacy in cell culture and in animal models. Their translation into therapeutics suitable for humans will depend upon cost, formula- tion, a favorable toxicity profile and a convenient dosing scheme. This approach is likely to be of particular importance in the battle against rapidly emerging infectious diseases, such as SARS or avian influenza.

1. Palliser, D. et al. Nature, 439, 89-94 (2006).

2. Elbashir, S.M. et al. Nature 411, 494-498 (2001).

3. Bitko, V., Musiyenko, A., Shulyayeva, O.\& Barik, S. Nat. Med. 11, 50-55 (2005).

4. Zhang, W. et al. Nat. Med. 11, 56-62 (2005).

5. Li, B.J. et al. Nat. Med. 11, 944-951 (2005).

6. Tompkins, S.M., Lo, C.Y., Tumpey, T.M. \& Epstein, S.L. Proc. Natl. Acad. Sci. USA 101, 8682-8686 (2004).

7. Ge, Q., Eisen, H.N. \& Chen, J. Virus Res. 102, 37-42 (2004).

8. Wilson, J.A. \& Richardson, C.D. J. Virol. 79, 7050 7058 (2005).

9. Gitlin, L., Stone, J.K. \& Andino, R. J. Virol. 79 1027-1035 (2005).

10. Boden, D., Pusch, O., Lee, F., Tucker, L. \& Ramratnam, B. J. Virol. 77, 11531-11535 (2003). 